\title{
Acanthamoeba keratitis associated with contact lenses: six consecutive cases of successful management
}

\author{
MARY BETH MOORE AND JAMES P McCULLEY \\ From the Department of Ophthalmology, University of Texas Southwestern Medical Center at Dallas, Texas, \\ USA
}

SUMMARY We examined and treated six patients with acanthamoeba keratitis associated with contact lens wear from 1981 to 1988 . Five patients were treated with topical neomycin-polymyxin B-gramicidin (Neosporin) and propamidine isethionate (Brolene) drops. The patients were followed up for an average of 32 months (range 16-75 months). Two patients underwent penetrating keratoplasty at 22 and 26 months after the onset of symptoms and have maintained clear grafts with no evidence of recurrence. In four patients corneal infiltrates cleared on topical medication. All six patients have $6 / 6$ best corrected vision. Early diagnosis and medical treatment alone can result in resolution of corneal infiltrates due to acanthamoebae. With this initial therapy we have had no treatment failures.

Acanthamoeba keratitis has become an increasingly important problem. ${ }^{12}$ A large majority of recently reported cases have occurred in contact lens wearers. ${ }^{34}$ Initially recognised in wearers of soft contact lenses, acanthamoeba keratitis is now being reported in wearers of hard lenses, wearers of gaspermeable hard lenses and of Saturn II lenses. It is now well established that the probable source of the infection is contaminated home-made saline or tap water rinse. Efforts are now underway to educate colleagues and patients about the potential risks of these sources of infection.

Much knowledge has been gained about how to diagnose this infection rapidly, and therefore infections are now being diagnosed very early in their course. We have been using propamidine isethionate and neomycin-polymyxin B-gramicidin since 1984 , when it was originally suggested to us by Peter Wright, and have had no treatment failures. While isolated cases have been successfully treated on a medical regimen alone, this is the first consecutive series of patients with acanthamoeba keratitis successfully treated by this regimen.

\section{Case reports}

CASE 1

This 13-year-old female, seen in December 1981, had

Correspondence to Mary Beth Moore, MD, UT Southwestern Ophthalmology, 5323 Harry Hines Blvd, Dallas, Texas 75235-9057, USA. an irritated right eye for one month. She wore dailywear soft contact lenses for myopia. She made homemade saline from salt tablets and distilled water, and chemically disinfected the lenses every one to two weeks. She was treated with antibiotic drops by her referring ophthalmologist, who noted subepithelial opacities, a preauricular node, and a mild follicular conjunctivitis.

On our initial examination the visual acuity was $6 / 60$ right eye and 6/6 left eye. There was marked epithelial mottling inferiorly without specific dendrite formation, underlying moderate stromal oedema, scattered nummular lesions in the anterior stroma both superiorly and inferiorly, and moderate keratic precipitates on the endothelium. The corneal sensation was decreased and there was moderate flare in the anterior chamber. The initial clinical impression was of herpes simplex keratitis. Over the next few months a ring infiltrate developed centrally with an overlying epithelial defect, leading to rapid melting of the anterior stroma. Six months later the cornea had become totally vascularised. In July 1983, 22 months after her initial symptoms, a penetrating keratoplasty was done to prevent perforation through a descemetocele.

Histological examination of the corneal button showed chronic granulomatous inflammation in the area of the ring infiltrate. No organisms were seen. Since April 1988 the patient has had a clear, compact graft, a best corrected visual acuity of $6 / 6$ with a hard contact lens, and no clinical evidence of recurrence in 
the graft 4 years and 9 months after surgery.

Acanthamoeba keratitis was not suspected in this patient until 1984, when case 2 was diagnosed. The corneal button was re-examined; however, no cysts or trophozoites were found. Indirect immunofluorescent antibody staining was positive for A. castellanii. Saline solutions and contact lenses were not available for culture.

CASE 2

This 30-year-old female presented in July 1984 with a one-month history of presumed herpes simplex keratitis. She wore extended-wear soft contact lenses and made her own saline solution from salt tablets and distilled water. The lenses were removed every two weeks, cleaned with the saline solution, then reinserted.

Her visual acuity was 6/90 right eye and 6/6 left eye. The right cornea was oedematous. Anterior stromal infiltrates were concentrated over the visual axis and extending nasally in a dendritiform pattern. The corneal sensation was decreased, and there were a few keratic precipitates and only minimal cell and flare. She was treated for a presumed herpes simplex stromal keratitis.

One month later a dense anterior stromal ring infiltrate and a radial nerve infiltrate developed. Neomycin-polymyxin B-gramicidin four times a day was begun for suspected acanthamoeba keratitis. The ring infiltrate improved on this regimen. Two months later cultures of the cornea were positive for acanthamoebae. At Peter Wright's suggestion propamidine isethionate $1 \%$ (Brolene) was begun four times a day. Idoxuridine was stopped and the steroids were tapered. There was dramatic improvement in the ring infiltrate, and the vision improved to $6 / 24$ with pinhole. All medications were stopped in March 1986, and by August there were no signs of recurrence. A penetrating keratoplasty was done 26 months after her initial symptoms. No trophozoites or cysts were seen in the corneal button with haematoxylin and eosin, periodic-acid-Schiff, and methenamine silver staining. However, immunofluorescent antibody staining was used to identify A. castellanii. Since April 1988 this patient has had a clear corneal transplant, a best corrected visual acuity of $6 / 6$, and no evidence of recurrence in the graft, 20 months after surgery.

CASE 3

This 23-year-old female was seen in September 1986 with a four-day history of itching, burning, and mucous discharge from the left eye. She wore extended-wear soft contact lenses for two weeks at a time and cleaned them with a commercially prepared cleaner and enzyme tablets dissolved in a commer- cially prepared saline. She chemically disinfected the lenses once a month and cleaned the lenses with the enzymatic cleaner once a month. She alternated these two procedures so that each one was done once every two months. Immediately before putting the contact lenses in her eyes, she rinsed them in tap water.

The visual acuity with pinhole was $6 / 12$ both eyes. There were multiple, hazy, grey-white anterior stromal infiltrates in the mid periphery of the left cornea in a semicircular pattern. The cornea was routinely cultured and the patient was treated for bacterial keratitis. Two days later there was a marked increase in the size and density of the stromal infiltrates. Acanthamoeba keratitis was suspected. However, because the epithelium was intact and the infiltrates deep, a corneal biopsy was done.

Smears from the base of the biopsy showed multiple, poorly staining, trophozoites on Gram and Geimsa stains. Histological examination of the biopsy gave negative results when examined with haematoxylin and eosin, methenamine silver, and periodic-acid-Schiff stains. Calcofluor white stain showed a few cysts on the posterior border of the biopsy specimen. The patient was started on propamidine isethionate and neomycin-polymyxingramicidin every 15 minutes and was admitted to the hospital for round-the-clock therapy. She stayed in the hospital three days and was then discharged on hourly therapy while awake and every two-hourly during the night. Two weeks later the corneal infiltrates had completely cleared, leaving faint scars.

Brolene and Neosporin were continued for one year four times a day initially and then gradually tapered to twice a day. Since April 1988 her cornea has been clear except for a scar at the biopsy site. The vision is $6 / 6$ with spectacles. She had been off medication for seven months and there is no sign of recurrence. There has been no sign of active infection for 17 months.

\section{CASE 4}

This 25-year-old male presented in September 1986 with a history of pain, redness, watering, and a foreign body sensation in the right eye. He wore extended-wear soft contact lenses.

His visual acuity was $6 / 6$ in both eyes. Examination of the cornea showed multiple tiny anterior stromal corneal infiltrates. The anterior chamber was deep and quiet and no other abnormalities were found. He was treated for presumed bacterial keratitis. However, one week later the infiltrates had progressed to diffuse patchy greyish white anterior stromal infiltrates in a semicircular pattern. The epithelium was intact and there was $2+$ cell and flare in the anterior chamber. 
Additional history revealed that during a camping trip his contact lens dried out and he had rehydrated it with well water and tap water on two separate occasions. A presumptive diagnosis of acanthamoeba keratitis was made based on the history of exposure to non-sterile solutions and the appearance of the corneal infiltrates, which were identical to the infiltrates in case 3. Propamidine isethionate and neomycin-polymyxin-gramicidin were started every 15 minutes and he was admitted to the hospital for three days of round-the-clock therapy. Three days later the drops were tapered to every 30 minutes while awake and every hour while asleep.

The drops were then slowly tapered to four times a day and then over the next 12 months tapered to twice a day and stopped. Since April 1988 he has continued to have $6 / 6$ vision with spectacles. He has a clear cornea with some faint residual scarring in the mid periphery and has been off all medication for seven months, with no sign of recurrence. There has been no sign of active infection for the past 17 months.

\section{CASE 5}

This 31-year-old female presented in October 1986 with a one-week history of superficial keratitis in the left eye, unresponsive to topical antibiotics. Four weeks prior to that she had been switched from her soft contact lenses to Saturn II lenses. She used tap water which had been filtered through a micropore filter system to make her homemade saline. The visual acuity was 6/6 right eye and 6/24 left eye. Examination of the left cornea revealed a central patch of mottled, somewhat opaque, epithelium resembling confluent toxic epitheliopathy. The underlying stroma was clear, and there was moderate cell and flare in the anterior chamber. The remainder of the examination of both eyes was normal.

The initial clinical impression was presumed toxicity from either hydrogen peroxide or thiomersal (thimerosal). She was treated with antibiotic ointment and pressure patching. Two days later the epitheliopathy had increased and there were inflammatory cells in the anterior stroma. The cul-de-sac and cornea were cultured for acanthamoebae. The cultures from the cornea, the contact lens solutions, and the filtered tap water were positive for acanthamoebae. The cul-de-sac culture was negative. Propamidine isethionate and neomycinpolymyxin-gramicidin were started every hour round the clock.

Two weeks later the eye was white and the cornea was clear. The medications were gradually decreased to four times a day and then tapered over the next 12 months and stopped. Since April 1988 she has been off all medication for six months and has had no evidence of recurrence. There has been no sign of active infection for 16 months. The visual acuity is $6 / 6$ with spectacles.

CASE 6

This 32-year-old female had been treated for herpes simplex keratitis in the left eye by her first ophthalmologist after developing symptoms in January 1987. When the keratitis did not improve, she was seen by a second ophthalmologist, who debrided the cornea twice and placed a bandage lens on the eye. When the keratitis did not improve with this regimen, she was referred to one of us.

The patient had worn hard contact lenses for many years. She stored her contacts in a home-made saline solution made from distilled water and salt tablets. Her jug of distilled water had been kept under the sink for at least two years. She soaked the lenses overnight in the home-made saline and washed them with a commercial cleaning preparation in the morning. She would then rinse them again with the home-made saline prior to insertion.

On examination in March her visual acuity was 6/6 right eye and $6 / 60$ left eye. Centrally there was a large area of anterior stromal opacification. Within this area there were several nummular, denser anterior stromal infiltrates. There was a corneal nerve infiltrate at 7 o'clock.

The initial diagnosis of acanthamoeba keratitis was based on the radial nerve infiltrate and the history of home-made saline solutions. Since the epithelium was intact, the cornea was not scraped or cultured. Propamidine isethionate and neomycin-polymyxingramicidin were started every hour while she was awake. Cultures of the cul-de-sac were negative for acanthamoebae; however, cultures of the contact lens case and home-made saline were positive.

One month later the infiltrate had completely resolved, leaving a central corneal scar. The drops were decreased to four times a day and then tapered over the next 12 months. Since April 1988 her best corrected vision was $6 / 6$ with a hard contact lens, and there has been no sign of recurrence or active infection for the past 16 months.

\section{Discussion}

Fortunately acanthamoeba keratitis is not rapidly progressive in comparison with some bacterial ulcers, and therefore it gives the practitioner a chance to intervene successfully even if symptoms and signs have been present for one to two weeks, or even one month. Intervening at the 1-4-week stage with topical propamidine isethionate and neomycinpolymyxin-gramicidin has proved to be effective in eliminating corneal infiltrates and maintaining a clear 
Table 1 Patient data

\begin{tabular}{|c|c|c|c|c|c|c|}
\hline Case & Age/sex & $C L$, solutions & Initial diagnosis & Treatment & $B C V A$ & $\begin{array}{l}\text { Follow-up } \\
\text { (months) }\end{array}$ \\
\hline 1 & $13 \mathrm{~F}$ & DWSCL, ST and DW & Herpes simplex keratitis & $\begin{array}{l}\text { Steroids, antivirals, antibiotics, PK at } 22 \\
\text { months }\end{array}$ & $6 / 6$ & 75 \\
\hline 2 & $30 \mathrm{~F}$ & EWSCL, ST and DW & Herpes simplex keratitis & $\begin{array}{l}\text { Steroids, antivirals, antibiotics. Brolene and } \\
\text { Neosporin at } 5 \text { months, PK at } 26 \text { months }\end{array}$ & $6 / 6$ & 46 \\
\hline 3 & $23 \mathrm{~F}$ & $\begin{array}{l}\text { EWSCL, commercial } \\
\text { saline, TWR }\end{array}$ & Bacterial keratitis & Brolene and Neosporin at 1 week & $6 / 6$ & 20 \\
\hline 4 & $25 \mathrm{M}$ & EWSCL, WW, TW & Bacterial keratitis & Brolene and Neosporin at 1 week & $6 / 6$ & 20 \\
\hline 5 & $31 \mathrm{~F}$ & Saturn II Lens, ST and TW & Bacterial keratitis & Brolene and Neosporin at 10 days & $6 / 6$ & 19 \\
\hline 6 & $32 \mathrm{~F}$ & HCL, ST and DW & Herpes simplex keratitis & $\begin{array}{l}\text { Steroids, antivirals, antibiotics. Brolene and } \\
\text { Neosporin at } 3 \text { months }\end{array}$ & $6 / 6$ & 16 \\
\hline
\end{tabular}

DWSCL = daily-wear soft contact lens. $E W S C L=$ extended-wear soft contact lens. $\mathrm{HCL}=$ hard contact lens. $\mathrm{ST}=$ salt tablets. $\mathrm{DW}=$ distilled water. $T W=$ tap water. $T W R=$ tap water rinse. $W W=$ well water. $P K=$ penetrating keratoplasty. $B C V A=$ best corrected visual acuity. Brolene (propamidine isethionate); Neosporin (neomycin-polymyxin-gramicidin).

cornea and useful vision in our series. Our results agree with those of earlier isolated case reports of successful medical treatment. ${ }^{56}$

Helpful clinical signs and history which may suggest an early acanthamoeba keratitis include the following: (1) previous treatment for herpes simplex heratitis with no improvement; (2) contact lens wear with exposure to home-made solution, tap water rinse, well water, intravenous saline; (3) bulbar injection but little chemosis and no discharge, mild to moderate lid oedema; (4) pain out of proportion to the clinical fihdings, infiltrates along the corneal nerves'; (5) epithelial irregularity or vesicular appearance in a pseudodendritic or linear pattern, epithelial haze, spherules, cystic lesions, refractile bodies, or recurrent epithelial erosions; (6) $0.5 \mathrm{~mm}$ diameter, white, discrete anterior stromal opacities, either single or multiple; (7) patchy anterior stromal greyish white infiltrates, multiple and variable in size, 1-3 $\mathrm{mm}$ with indistinct edges, forming a crescent or semicircular pattern; (8) stromal ring infiltrate or partial ring infiltrate (crescent).

Our initial treatment is intensive, and includes topical neomycin-polymyxin-gramicidin and propamidine isethionate every 15 minutes to every hour round the clock for three days, then every hour while the patient is awake and every two hours while asleep for three days, and then every hour while awake for another week. The drops are then tapered until a four times a day maintenance dose can be maintained. All patients on intensive topical medication will show signs of presumed drug toxicity. These include punctate epithelial erosions, an increase in bulbar conjunctival injection and chemosis, and a follicular response in the upper and lower tarsal conjunctival surfaces. This toxic response is reversible and should not influence or change the medical regimen.

Preliminary studies in our laboratory indicate that pentamidine and propamidine isethionate are amoebacidal in vitro and confirm previous study results. ${ }^{5-10}$ Our rationale in the initial treatment of this keratitis is to saturate the cornea with the drug in an effort to kill trophozoites, to encourage encystment, and to maintain a drug level high enough to kill any emerging trophozoites should the cysts survive and later cause recurrence of the keratitis. We consider that delaying aggressive topical therapy could theoretically result in a significant increase in the number of organisms. A cornea which has been infected for 2-3 months may contain so many organisms that it is simply not possible to eradicate all of them with topical drops four times a day. This may account for some cases of reported failure of medical treatment."

We do not use topical steroids because we consider that host defences are important in eradicating the organism and steroids inhibit neutrophil and macrophage response. The initial in-vitro study which reported that steroids inhibited morphogenesis of acanthamoeba organisms was not substantiated in subsequent animal studies ${ }^{12}$ (Osato $M$, personal communication 1987). We therefore believe that inhibition of morphogenesis would probably not take place in the clinical situation and steroids should not be used in an effort to accomplish this.

Two of our patients underwent penetrating keratoplasty. The first patient was diagnosed in retrospect by examining the corneal button. This patient never received specific antiamoebic therapy, and so we do not consider this a medical failure. The surgery was performed 22 months after the presumed initial infection and was done for a threatening corneal perforation. By this time the keratitis appeared walled off, on both slit-lamp and histological examination, and therefore we do not consider this a surgical cure but rather a surgical solution to an optical and mechanical problem. 
The second patient was misdiagnosed and treated with steroids, antivirals, and antibiotics for five months before the correct diagnosis was made. Specific antiamoebic therapy, propamidine isethionate and neosporin-polymyxin-gramicidin, was used for 15 months. Six months later, after no sign of recurrence off specific therapy, a penetrating keratoplasty was performed for optical rehabilitation, not therapeutic reasons. We consider this a case of successful medical treatment because the infection was arrested and controlled with specific topical medication and because there was no recurrence either before or after surgery when specific medication was discontinued.

We have shown that we have achieved consistently good results with this approach even though patients were treated at different times from the onset of their symptoms (Table 1). These results were achieved owing to a high level of suspicion and early appropriate medical therapy. We strongly recommend using this approach initially rather than resorting to a corneal transplant in an eye with an uncontrolled infection.

We thank Peter Wright, who has been a constant source of information and guidance on the medical treatment of acanthamoeba keratitis.

This work was supported in part by an unrestricted research grant from Research to Prevent Blindness, Inc, New York, New York.

\section{References}

1 Morbidity and Mortality Weekly Report. Acanthamoeba keratitis associated with contact lenses-United States. Centers for Disease Control, 27 June, 1986: 35: no. 25.

2 Ayran JD, Starr MB, Jakobiec FA: Acanthamoeba keratitis: a review of the literature. Cornea 1987; 6: 2-26.

3 Moore MB, McCulley JP, Luckenbach M, et al. Acanthamoeba keratitis associated with soft contact lenses. Am J Ophthalmol 1985; 100: 396-403.

4 Moore MB, McCulley JP, Newton C, et al. Acanthamoeba keratitis; a growing problem in hard and soft contact lenses wearers. Ophthalmology 1987; 94: 1654-61.

5 Wright $\mathrm{P}$, Warhurst $\mathrm{D}$, Jones BR. Acanthamoeba keratitis successfully treated medically. Br J Ophthalmol 1985; 69: 77882.

6 Jackson TN, Heinze JB, Tuxen J, Weiner JM. Successful medical treatment of a corneal ulcer due to Acanthamoeba polyphaga. Aust NZ J Ophthalmol 1986; 14: 139-42.

7 Moore MB, McCulley JP, Kaufman HE, Robin JB. Radial keratoneuritis as a presenting sign in acanthamoeba keratitis. Ophthalmology 1986; 93: 1310-5.

8 Casemore DP. Sensitivity of hartmannella (acanthamoeba) to 5-fluorocystosine, hydroxystilbamidine, and other substances. J Clin Pathol 1970; 23: 649-52.

9 Nagington J, Richards JE. Chemotherapeutic compounds and acanthamoeba from eye infections. J Clin Pathol 1976; 29: 64851.

10 Ferrante A, Rowan-Kelly B, Thong YH. In vitro sensitivity of virulent Acanthamoeba culbertsoni to a variety of drugs and antibiotics. Int J Parasitol 1984; 14: 53-6.

11 Moore MB, McCulley JP. Letter to the editor. Am J Ophthalmol 1987; 104: 310-1.

12 Osato M, Robinson N, Wilhelmus K, Jones D. Morphogenesis of Acanthamoeba castellanii: titration of the steroid effect. Invest Ophthalmol Vis Sci 1986; 27: 37.

Accepted for publication 19 May 1988. 